\title{
Editorial
}

\section{Stability Analysis Including Monostability and Multistability in Dynamical System and Applications}

\author{
Zhenkun Huang, ${ }^{1}$ Anke Meyer-Baese, ${ }^{2}$ Chunhua Feng, ${ }^{3}$ P. Balasubramaniam, ${ }^{4}$ Kelin Li, ${ }^{5}$ \\ and Eva Kaslik ${ }^{6}$ \\ ${ }^{1}$ School of Science, Jimei University, Xiamen 361021, China \\ ${ }^{2}$ Department of Electrical Engineering, Florida State University, 2525 Pottsdamer Street, Tallahassee, FL 32310-6046, USA \\ ${ }^{3}$ Department of Mathematics, Guangxi Normal University, Guangxi 541004, China \\ ${ }^{4}$ Department of Mathematics, Gandhigram Rural Institute-Deemed University, Gandhigram, Tamil Nadu 624 302, India \\ ${ }^{5}$ Department of Mathematics, Sichuan University of Science \& Engineering, Sichuan 643000, China \\ ${ }^{6}$ Department of Mathematics and Computer Science, West University of Timisoara, Boulevard Corneliu Coposu, 43300223 Timisoara, \\ Romania
}

Correspondence should be addressed to Zhenkun Huang; hzk974226@jmu.edu.cn

Received 3 December 2013; Accepted 3 December 2013; Published 27 January 2014

Copyright (C) 2014 Zhenkun Huang et al. This is an open access article distributed under the Creative Commons Attribution License, which permits unrestricted use, distribution, and reproduction in any medium, provided the original work is properly cited.

Stability theory has been significantly developed and extended to dynamical system which will provide a deep insight into a more comprehensive understanding of the dynamic structure of complex dynamical systems and therefore is of great significance for its application. The overall aim of this special issue is to open a discussion among researchers actively working in the field of stability theory and relative applications. The issue covers a wide variety of problems for neural networks and applications such as image processing and pedestrian detection, difference equations, dynamic equations on time scales, biological mathematics including multigroup and reaction-diffusion epidemic model, and soft computing. In the following, we briefly review each of the papers by highlighting the significance of the key contributions.

In "Periodic oscillation analysis for a coupled FHN network model with delays" by Y. Lin, the author provides new results of periodic oscillatory behavior of three coupled FHN neurons model by Chafee's criterion of limit cycle. In "Dynamical behaviors of the stochastic hopfield neural networks with mixed time delays" by $\mathrm{L}$. Wan et al., the authors of this paper investigate dynamical behaviors of the stochastic Hopfield neural networks with mixed time delays.
By employing the theory of stochastic functional differential equations and linear matrix inequality (LMI) approach, some novel criteria on asymptotic stability, ultimate boundedness, and weak attractor are derived.

Two papers in our special issue are devoted to discuss applications of neural networks. In "Analysis of feature fusion based on HIK SVM and its application for pedestrian detection" by S.-Z. Su and S.-Y. Chen, the author adopt support vector machine (SVM) with the histogram intersection kernel (HIK) as a classifier to detect pedestrians in lowresolution visual images and at night time. In "Recursive neural networks based on PSO for image parsing" by G.-R. Cai and S.-L. Chen, the authors give an image parsing algorithm based on Particle Swarm Optimization (PSO) and Recursive Neural Networks (RNNs).

A theoretical article titled " $L \omega$-compactness in $L \omega$-spaces" presents some important properties of $L \omega$-compactness. The authors, S.-L. Chen and J.-L. Huang, reveal the Alexander subbase lemma and the Tychonoff product theorem with respect to $L \omega$-compactness.

Six papers are concerned about dynamical analysis of difference equations or dynamic equations on time scales. In "Oscillation for higher order dynamic equations on time scales" 
by T. Sun et al., the authors present sufficient conditions to ensure every solution of higher order dynamic equations on time scales to be oscillatory or tend to zero. In " $h$-Stability for differential systems relative to initial time difference" by $\mathrm{P}$. Wang and X. Liu, the authors discuss $h$-stability for differential systems with initial time difference and stability criteria are formulated by using variation of parameter techniques. In "Periodic solutions of second-order difference problem with potential indefinite in sign" by $\mathrm{H}$. Bin, the author obtains some new results concerning the existence of nontrivial periodic solution of second-order difference problem with potential indefinite in sign by using Morse theory. In "Dynamics of a family of nonlinear delay difference equations" by Q. He et al., the authors give sufficient conditions guaranteeing the globally asymptotical stability of a unique positive equilibrium of nonlinear delay difference equations. In "Subharmonics with minimal periods for convex discrete hamiltonian systems" by $\mathrm{H}$. Bin, by using variational methods and dual functional, the author considers the subharmonics with minimal periods for convex discrete Hamiltonian systems. In "Leader-following consensus stability of discrete-time linear multiagent systems with observer-based protocols" by B. Xu et al., the authors obtain two types of distributed observer-based consensus protocols to solve the leader-following consensus problem of discrete-time multiagent systems on a directed communication topology.

There are two new results about epidemic models in this special issue.

In "Stability analysis of a multigroup epidemic model with general exposed distribution and nonlinear incidence rates" by L. Zhang et al., the authors adopt Lyapunov functionals and a graph-theoretical technique to derive sufficient conditions ensuring the global dynamics. In "Traveling wave solutions in a reaction-diffusion epidemic model" by $\mathrm{S}$. Wang et al., the authors investigate a unique and strictly monotonic traveling wave solutions in a reaction-diffusion epidemic model through monotone iteration of a pair of classical upper and lower solutions.

\title{
Acknowledgments
}

The guest editors of this special issue would like to express their thanks to the authors who have submitted papers for consideration and the referees of the submitted papers.

\author{
Zhenkun Huang \\ Anke Meyer-Baese \\ Chunhua Feng \\ P. Balasubramaniam \\ Kelin Li \\ Eva Kaslik
}




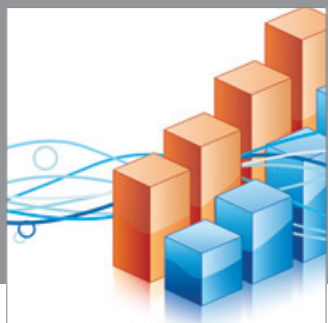

Advances in

Operations Research

mansans

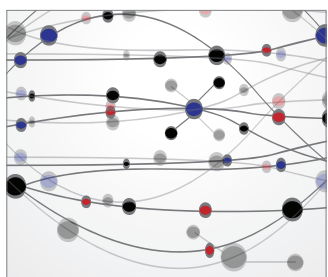

The Scientific World Journal
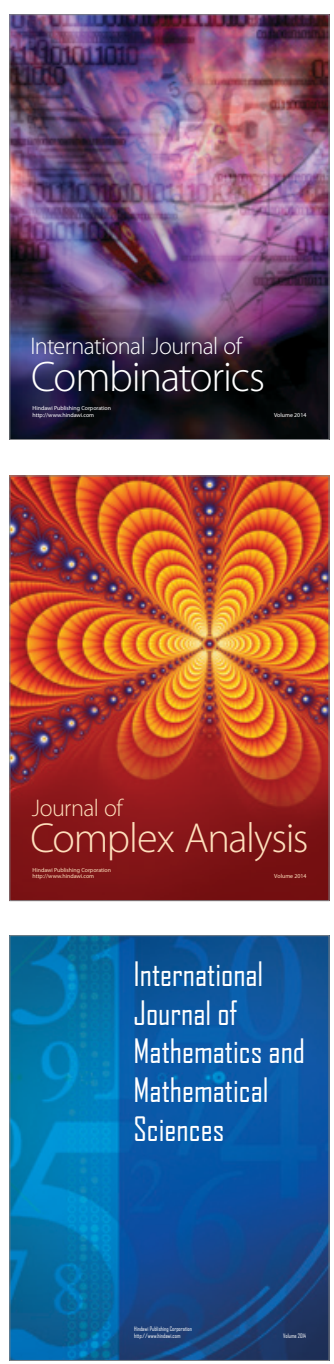
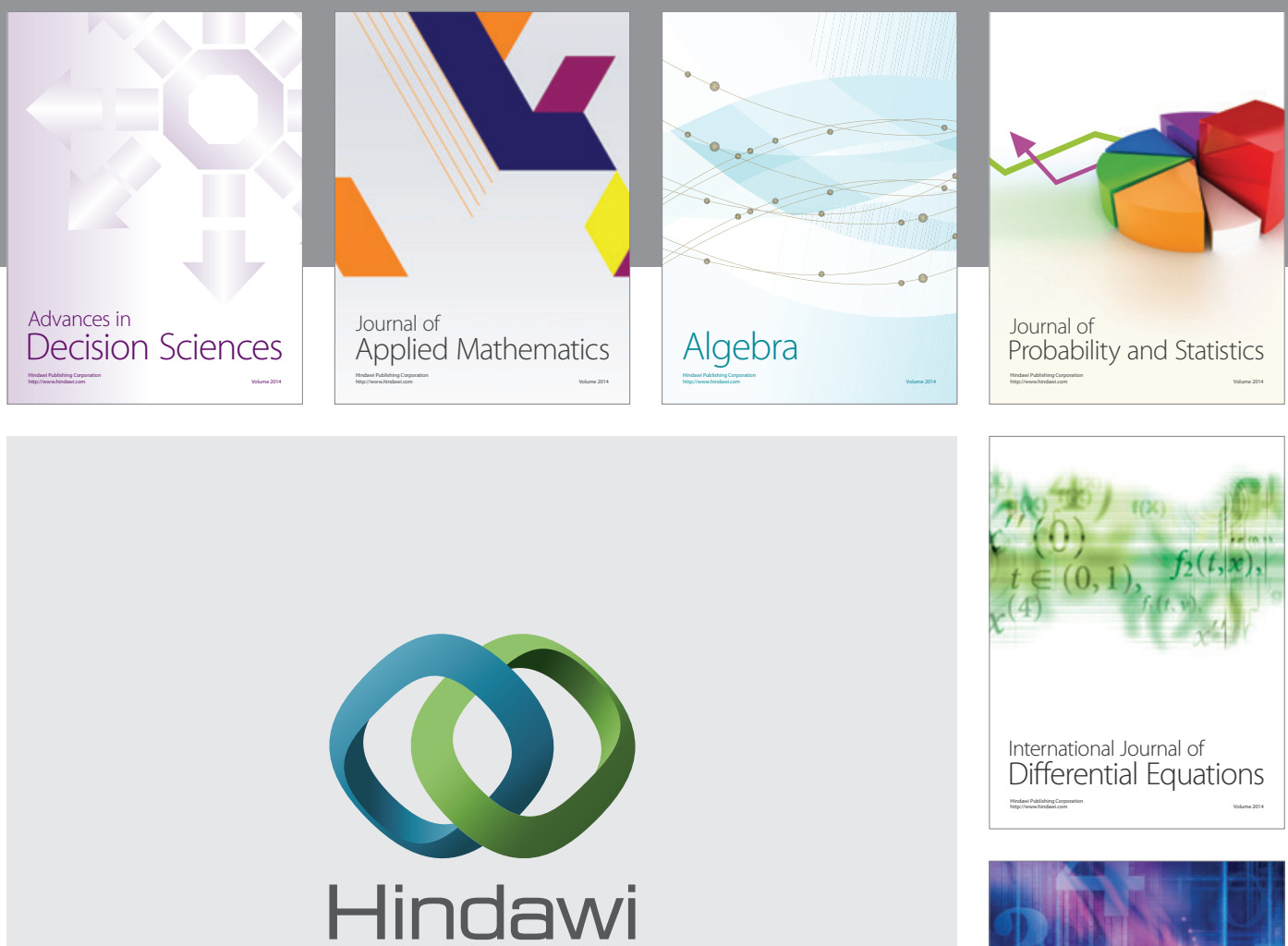

Submit your manuscripts at http://www.hindawi.com
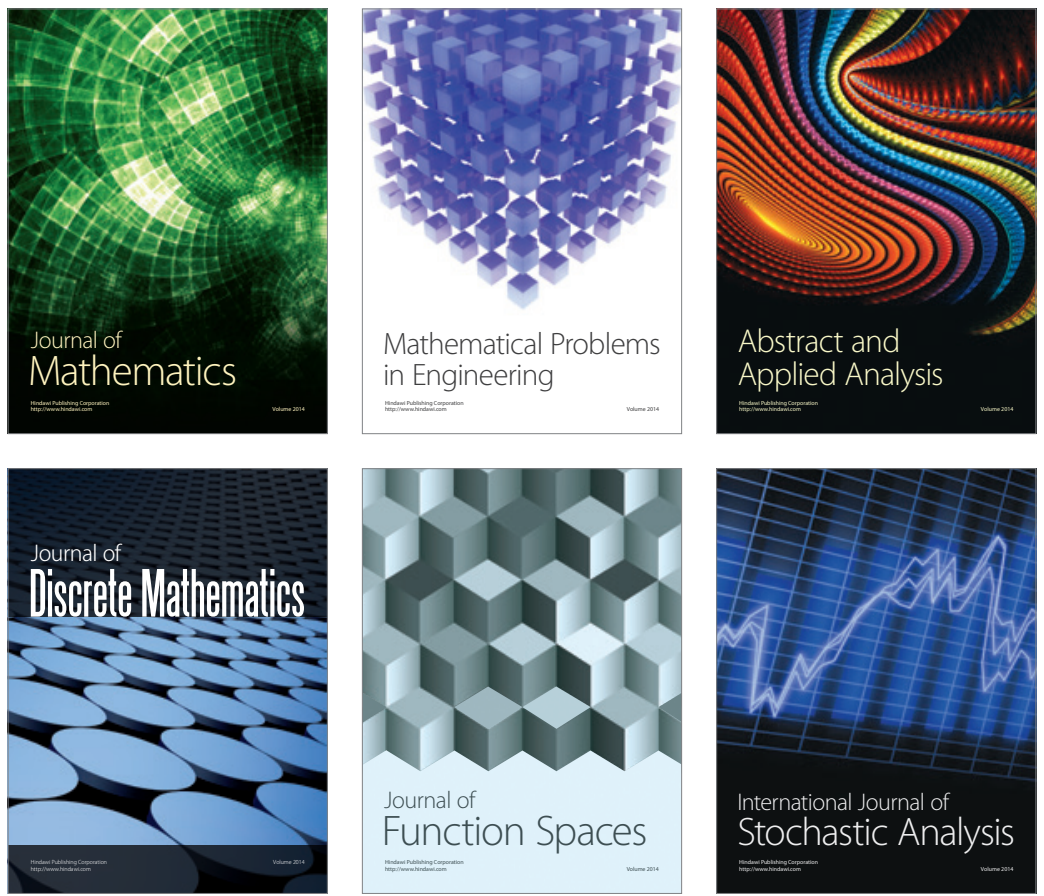

Journal of

Function Spaces

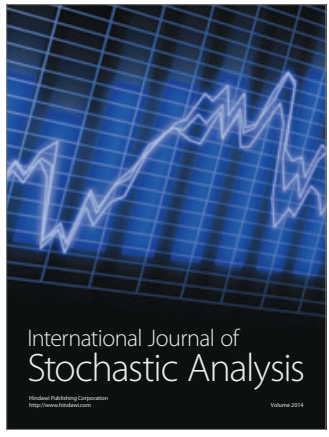

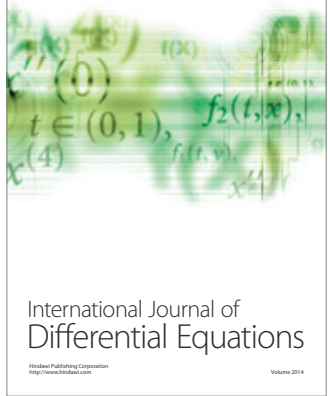
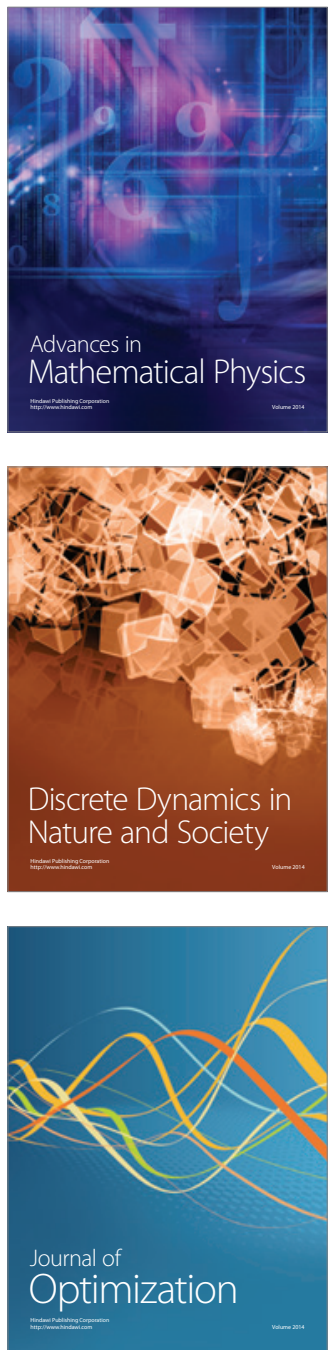